\title{
¿SE PUEDE REDUCIR EL ÍNDICE DE CESÁREA? EXPERIENCIA DEL HOSPITAL CLÍNICO DE LA UNIVERSIDAD DE CHILE*
}

\author{
Drs. Hugo Salinas $P^{1}$, Sergio Carmona G. ${ }^{1}$, Jaime Albornoz $V^{1}{ }^{1}$, Patricio Veloz $R^{2 a}$, \\ Rodrigo Terra V.' , Rubén Marchant G. ${ }^{1}$, Verónica Larrea E. ${ }^{1}$, Rodrigo Guzmán R.', \\ Luis Martínez M. ${ }^{1}$
}

${ }^{1}$ Departamento de Obstetricia y Ginecología. ${ }^{2}$ Gerencia de Operaciones, Hospital Clínico J.J. Aguirre, Universidad de Chile.

alngeniero Civil.

\section{RESUMEN}

Se presenta la experiencia de la Maternidad del Hospital Clínico de la Universidad de Chile de un programa diseñado especialmente con el objetivo de disminuir las cesáreas evitables. Tomando el porcentaje de cesáreas del trimestre enero-marzo de 2003 como base. Se efectuó un programa de intervención entre los meses de abril-septiembre de 2003, que considera: entrega diaria del turno de residencia, segunda opinión para operación cesárea, diagnóstico pormenorizado de pelvis en ficha clínica, auditoría mensual de cesáreas "evitables" y estandarización de un protocolo de presentaciones distócicas. El propósito del estudio fue evaluar el efecto de la introducción de un sistema de auditoría en la reducción de la tasa de cesáreas, según propuesta de Robson y cols. El porcentaje global de cesáreas disminuyó de $44,9 \%$ a $37,1 \%$, en nulíparas de $39,0 \%$ a $29,5 \%$ y en multíparas de $48,1 \%$ a $42,2 \%$, diferencias estadísticamente significativas.

\section{PALABRAS CLAVE: Cesáreas, gestión clínica, auditoría médica, efecto Hawthorne}

\section{SUMMARY}

The experience at the Maternity Ward of Hospital Clínico de la Universidad de Chile in reducing avoidable cesarean sections is presented. The purpose of this study is to analyze the effects of an audit cycle in the cesarean section rate, using the proposal of Robson et al.

A specifically designed program is used. Using the cesarean section rate of january to march trimester as a baseline, we designed an intervention protocol for april to september period which involves: daily resident report, second opinion for cesarean section, detailed pelvis diagnosis in the medical file, monthly "avoidable" cesarean section audit cycle and abnormal lie protocol standardization.

The overall cesarean section rate was reduced from $44.9 \%$ to $37.1 \%$, in nulliparous from $39.0 \%$ to $29.5 \%$ and in multiparous from $48.1 \%$ to $42.2 \%$, all these differences were statistically significant.

KEY WORDS: Cesarean section, clinical management, medical audit, Hawthorne effect

\footnotetext{
*Trabajo leído en la sesión del martes 16 de diciembre de 2003 de la Sociedad Chilena de Obstetricia y Ginecología.
} 


\section{INTRODUCCIÓN}

Las tasas de cesáreas han aumentado de manera importante en los últimos años y las razones no son del todo explicables (1). Si bien existen diferencias geográficas, en Latinoamérica el aumento de las tasas es independiente de las causas (2). Es motivo de controversia la relación entre el aumento de la tasa de operación cesárea y los índices de morbilidad y mortalidad perinatal, manifiestándose aún dudas acerca de los potenciales efectos adversos de la intervención, la mayoría de los estudios apuntan a un efecto real en las siguientes gestaciones, especialmente cuando estas se resuelven por la vía vaginal (3-6).

Los economistas de la salud están demandando una mejor comprensión de las razones para la decisión de una operación cesárea o la vía vaginal frente a un caso determinado, especialmente por las implicancias financieras que deben ser asumidas por las instituciones de salud (7). La práctica obstétrica influye en la tasa de cesáreas y la mayoría de las estrategias utilizadas por diferentes organizaciones de salud apuntan a controlar la toma de decisión individual para reducir el número de cesáreas potencialmente evitables, mediante distintas formas de auditoría y así intervenir y controlar la práctica obstétrica $(8,9)$.

La auditoría médica ha sido definida como el análisis sistemático y crítico del cuidado médico, incluyendo los procedimientos usados para el diagnóstico y tratamiento, el uso de los recursos, y los resultados obtenidos con el paciente, incluida la mejoría de su calidad de vida (10). El ciclo de auditoría médica persigue producir cambios relevantes y consecuentemente la mejoría de los cuidados de salud $(11,12)$.

Con el objeto de asegurar un proceso riguroso de auditoría médica, se deben establecer estándares clínicos respecto de la operación cesárea y compararlos con la práctica habitual, proponiendo las modificaciones que sean necesarias para lo-

Tabla I

\section{PROGRAMA DE INTERVENCION DE CESÁREAS}

- Entrega diaria de turno de residencia.

- Segunda opinión para decidir operación cesárea.

- Diagnóstico pormenorizado de pelvis en ficha clínica.

- Auditoría mensual de cesáreas "evitables".

- Estandarización de protocolo de presentaciones distócicas. grar su total cumplimiento, para mejorar la eficacia $y$ eficiencia de las actividades realizadas.

El propósito del estudio fue evaluar los efectos de la introducción de un sistema de auditoría sobre las tasas de cesáreas.

\section{MATERIAL Y MÉTODOS}

En abril de 2003, se introdujo en el Servicio de Obstetricia del Hospital Clínico de la Universidad de Chile, un sistema de auditoría que tendiera a la atención de un parto seguro para la madre y el recién nacido, con mínima intervención y máxima satisfacción (Tabla I). Con el objeto de asegurar una línea basal de la tasa de cesáreas, se realizó una auditoría retrospectiva a todos los partos atendidos en el Servicio entre el 1 de enero y el 31 de marzo de 2003, identificando las cesáreas que pudieron ser evitadas.

El número y tasa de cesáreas en grupos relevantes de mujeres fueron establecidos por los criterios que se muestran en las Tablas II y III, con el objeto de identificar los grupos que más contribuyen a los niveles de cesáreas reportados.

Para el análisis de auditoría se utilizó la propuesta de Robson y cols (13). La comparación de proporciones se efectuó con la prueba de chi cuadrado. Se consideró significativo un $p<0,05$.

\section{RESULTADOS}

La Tabla IV muestra una reducción de las cesáreas en el período abril-septiembre, con respecto al establecido como base, tanto en multíparas como nulíparas. El porcentaje de cesáreas disminuyó desde $44,9 \%$ a $37,1 \%$ en forma global, siendo esta disminución en nulíparas de 39,0\% a $29,5 \%$ y en multíparas de 48,1 a $42,2 \%$, todas las diferencias fueron estadísticamente significativas.

La Tabla $V$ muestra muestra una reducción de las cesáreas en el período abril-septiembre, con respecto a enero-marzo, en todas las categorías establecidas. Existió diferencia significativa en los

\section{Tabla II}

\section{ANÁLISIS POR CATEGORÍA Y GESTACIÓN}

- Todos los embarazos simples, en cefálica, de término.

- Todas las presentaciones distócicas y podálicas.

- Todos los nacimientos prematuros (<37 semanas), simples y en cefálica.

- Todos los embarazos múltiples. 
Tabla III

\section{ANÁLISIS POR PARIDAD, CATEGORÍA, CURSO Y GESTACIÓN}

\section{Nuliparas}

Embarazos simples, en cefálica, de término:

- Trabajo de parto espontáneo.

- Trabajo de parto inducido o cesárea antes del parto. Resto: Presentación podálica, embarazo múltiple, presentación distócica y parto prematuro.

\section{Multiparas}

Embarazos simples, en cefálica, de término:

- Con cicatriz de cesárea previa.

- Sin cicatriz de cesárea previa.

Resto: Presentación podálica, embarazo múltiple, presentación distócica y parto prematuro con o sin cicatriz de cesárea previa. grupos de nulíparas y multíparas con embarazo simple, en cefálica y de término. La mayor reducción corresponde a multíparas, con embarazo simple y feto en presentación cefálica, con embarazo de término, sin cicatriz de cesárea previa, en las cuales la disminución fue de $16,9 \%$ a $10,6 \%$.

La Tabla VI resume la diferencia de las cesáreas en el período abril-septiembre, con respecto a enero-marzo, en todas las pacientes, sin considerar la paridad. Hubo diferencia significativa en el grupo de embarazo simple, en cefálica y de término.

La Tabla VII muestra la reducción de las cesáreas en el período abril-septiembre, con respecto al establecido como base, en todas las categorías de paridad, curso y edad gestacional. La diferencia fue significativa en todos los casos, salvo en el

Tabla IV

ÍNDICES DE CESÁREAS ENERO-MARZO Y ABRIL-SEPTIEMBRE. TODAS LAS MUJERES

\begin{tabular}{lccccccc}
\hline & \multicolumn{3}{c}{ Enero - Marzo } & \multicolumn{3}{c}{ Abril - Septiembre } \\
Grupo de mujeres & Cesáreas & Total & $\%$ & Cesáreas & Total & $\%$ & $p$ \\
\hline Todas & 184 & 409 & 44,9 & 307 & 827 & 37,1 & $<0,003$ \\
Nulíparas & 55 & 141 & 39,0 & 97 & 329 & 29,5 & $<0,01$ \\
Multíparas & 129 & 268 & 48,1 & 210 & 498 & 42,2 & $<0,0001$ \\
\hline
\end{tabular}

Tabla V

NÚMERO DE CESÁREAS ENERO-MARZO Y ABRIL-SEPTIEMBRE POR CATEGORÍA

\begin{tabular}{|c|c|c|c|c|c|c|c|}
\hline \multirow[b]{2}{*}{ Categoría } & \multicolumn{3}{|c|}{ Enero - Marzo } & \multicolumn{4}{|c|}{ Abril - Septiembre } \\
\hline & Cesáreas & Total & $\%$ & Cesáreas & Total & $\%$ & $p$ \\
\hline Todas las mujeres & 184 & 409 & 44,9 & 307 & 827 & 37,1 & $<0,003$ \\
\hline $\begin{array}{l}\text { Embarazos simples, en cefálica, de } \\
\text { término }\end{array}$ & 150 & 358 & 41,9 & 257 & 696 & 36,9 & $<0,05$ \\
\hline Distocias de presentación & 16 & 16 & 100,0 & 37 & 38 & 97,4 & $<0,24$ \\
\hline $\begin{array}{l}\text { Prematuros, embarazos simple, en } \\
\text { cefálica }\end{array}$ & 19 & 36 & 52,8 & 37 & 77 & 48,1 & $<0,31$ \\
\hline Gemelares & 0 & 0 & - & 15 & 18 & 83,3 & - \\
\hline $\begin{array}{l}\text { Nulíparas, embarazo simple, en cefálica, } \\
\text { de término, espontáneo }\end{array}$ & 17 & 75 & 22,7 & 29 & 177 & 16,4 & $<0,09$ \\
\hline $\begin{array}{l}\text { Nulíparas, embarazo simple, en cefálica, } \\
\text { de término, inducido o cesárea sin } \\
\text { trabajo de parto }\end{array}$ & 27 & 46 & 58,7 & 42 & 108 & 38,9 & $<0,01$ \\
\hline $\begin{array}{l}\text { Nulíparas, de pretérmino, distocias de } \\
\text { presentación y embarazos múltiples } \\
\text { (todo incluido) }\end{array}$ & 13 & 20 & 65,0 & 26 & 44 & 59,1 & $<0,32$ \\
\hline $\begin{array}{l}\text { Multíparas, embarazo simple, en cefálica, } \\
\text { de término sin cesárea previa }\end{array}$ & 24 & 142 & 16,9 & 27 & 253 & 10,7 & $<0,006$ \\
\hline $\begin{array}{l}\text { Multíparas, embarazo simple, en cefálica, } \\
\text { de término con cesárea previa }\end{array}$ & 84 & 95 & 88,4 & 122 & 158 & 77,2 & $<0,01$ \\
\hline $\begin{array}{l}\text { Multíparas, embarazo múltiple, distocia } \\
\text { de presentación, de pretérmino con o sin } \\
\text { cesárea previa (todas incluidas) }\end{array}$ & 21 & 31 & 67,7 & 61 & 87 & 70,1 & $<0,58$ \\
\hline
\end{tabular}


Tabla VI

ÍNDICES DE CESÁREAS ENERO-MARZO Y ABRIL-SEPTIEMBRE. POR CATEGORÍA Y EDAD GESTACIONAL. NULÍPARAS Y MULTÍPARAS COMBINADAS

\begin{tabular}{lrrrrrrrr}
\hline & \multicolumn{3}{c}{ Enero - Marzo } & \multicolumn{3}{c}{ Abril - Septiembre } \\
Grupo de mujeres & Cesáreas & Total & $\%$ & Cesáreas & Total & $\%$ & $p$ \\
\hline Todas & 184 & 409 & 44,9 & 307 & 827 & 37,1 & $<0,003$ \\
Embarazo simple, cefálica, de término & 150 & 358 & 41,9 & 220 & 696 & 31,6 & $<0,05$ \\
Distocias de presentación & 16 & 16 & 100,0 & 37 & 38 & 97,4 & $<0,24$ \\
Embarazo simple, en cefálica, de pretérmino & 19 & 36 & 52,8 & 37 & 77 & 48,1 & $<0,31$ \\
Embarazos múltiples & 0 & 0 & - & 15 & 18 & 83,3 & - \\
Todas menos embarazos simples, en & 34 & 51 & 66,7 & 87 & 131 & 66,4 & $<0,44$ \\
cefálica de término & & & & & & & \\
\hline
\end{tabular}

Tabla VII

\section{ÍNDICES DE CESÁREAS ENERO-MARZO Y ABRIL-SEPTIEMBRE. POR PARIDAD, CATEGORÍA, CURSO Y EDAD GESTACIONAL. SOLO NULÍPARAS}

\begin{tabular}{|c|c|c|c|c|c|c|c|}
\hline \multirow[b]{2}{*}{ Grupo de mujeres } & \multicolumn{3}{|c|}{ Enero - Marzo } & \multicolumn{4}{|c|}{ Abril - Septiembre } \\
\hline & Cesáreas & Total & $\%$ & Cesáreas & Total & $\%$ & $p$ \\
\hline Todas las nulíparas & 55 & 141 & 39,0 & 97 & 329 & 29,5 & $<0,01$ \\
\hline $\begin{array}{l}\text { Nulíparas, embarazo simple, en cefálica, } \\
\text { de término }\end{array}$ & 42 & 121 & 34,7 & 71 & 285 & 24,9 & $<0,02$ \\
\hline $\begin{array}{l}\text { Nulíparas, embarazo simple, en cefálica, } \\
\text { de término, espontáneo }\end{array}$ & 17 & 75 & 22,7 & 29 & 177 & 16,4 & $<0,09$ \\
\hline $\begin{array}{l}\text { Nulíparas, embarazo simple, en cefálica, } \\
\text { de término, inducido o cesárea sin trabajo } \\
\text { de parto }\end{array}$ & 27 & 46 & 58,7 & 42 & 108 & 38,9 & $<0,01$ \\
\hline $\begin{array}{l}\text { Nulíparas, de pretérmino, distocias de } \\
\text { presentación y embarazos múltiples } \\
\text { (todo incluido) }\end{array}$ & 13 & 20 & 65,0 & 26 & 44 & 59,1 & $<0,32$ \\
\hline $\begin{array}{l}\text { Todas nulíparas, excepto embarazos simples, } \\
\text { en cefálica, de término, en trabajo de parto } \\
\text { espontáneo }\end{array}$ & 38 & 66 & 57,6 & 68 & 152 & 44,7 & $<0,08$ \\
\hline
\end{tabular}

grupo de las pacientes nulíparas, cursando un embarazo múltiple, de pretérmino y con distocia de presentación.

La Tabla VIII muestra la reducción de las cesáreas en el período abril-septiembre, con respecto al establecido como base, en todas las categorías de paridad, curso y edad gestacional. La diferencia fue significativa en todos los casos, salvo en el grupo de las pacientes multíparas, cursando un embarazo múltiple, con distocia de presentación, de pretérmino con o sin cesárea previa (todas incluidas).

\section{DISCUSIÓN}

El propósito de este estudio fue establecer un método que permitiera tomar las medidas necesa- rias para reducir las cesáreas consideradas por el equipo clínico como "evitables", estableciendo al mismo tiempo que grupos de mujeres contribuyen en mayor proporción al índice de cesáreas en la Maternidad del Hospital Clínico de la Universidad de Chile.

Se consideró que es útil realizar un análisis de las indicaciones de cesárea después que se ha identificado y clasificado la incidencia de la operación cesárea de acuerdo a factores como paridad, modalidad de inicio del trabajo de parto, presentación y edad gestacional. El uso de un análisis estandarizado de los índices de cesáreas como el propuesto por Robson y cols. (13), nos permitió comparar las tasas mes a mes, utilizando como base el período enero-marzo y el período de intervención abril-septiembre del mismo año. 
Tabla VIII

ÍNDICES DE CESÁREAS ENERO-MARZO Y ABRIL-SEPTIEMBRE. POR PARIDAD, CATEGORÍA, CURSO Y EDAD GESTACIONAL. SOLO MULTÍPARAS

\begin{tabular}{|c|c|c|c|c|c|c|c|}
\hline \multirow[b]{2}{*}{ Grupo de mujeres } & \multicolumn{3}{|c|}{ Enero - Marzo } & \multicolumn{4}{|c|}{ Abril - Septiembre } \\
\hline & Cesáreas & Total & $\%$ & Cesáreas & Total & $\%$ & $p$ \\
\hline Todas las multíparas & 129 & 268 & 48,1 & 210 & 498 & 42,2 & $<0,05$ \\
\hline $\begin{array}{l}\text { Multíparas, embarazo simple, cefálica, de } \\
\text { término }\end{array}$ & 108 & 237 & 45,6 & 149 & 411 & 36,3 & $<0,006$ \\
\hline $\begin{array}{l}\text { Multíparas, embarazo simple, en cefálica, de } \\
\text { término sin cesárea previa }\end{array}$ & 24 & 142 & 16,9 & 27 & 253 & 10,7 & $<0,04$ \\
\hline $\begin{array}{l}\text { Multíparas, embarazo simple, en cefálica de } \\
\text { término con cesárea previa }\end{array}$ & 84 & 95 & 88,4 & 122 & 158 & 77,2 & $<0,01$ \\
\hline $\begin{array}{l}\text { Multíparas, embarazo múltiple, distocia de } \\
\text { presentación, de pretérmino con o sin cesárea } \\
\text { previa (todas incluidas) }\end{array}$ & 21 & 31 & 67,7 & 61 & 87 & 70,1 & $<0,58$ \\
\hline
\end{tabular}

Este estudio describe la introducción de un ciclo de auditorías médicas en la práctica del Servicio de Obstetricia, logrando la reducción significativa de los índices de cesárea. En nuestra opinión la aplicación de la auditoría en la Residencia es apropiada, especialmente porque el equipo obstétrico permanece en su lugar de trabajo durante 12 a 24 horas, siendo fácil efectuar el seguimiento de los casos clínicos y lograr que los médicos asuman la responsabilidad por la segunda opinión prequirúrgica.

El cambio en el manejo obstétrico ha sido dirigido a las matronas y principalmente a los médicos, con énfasis en la identificación y tratamiento de las distocias y otros factores que contribuyen a la necesidad de practicar una cesárea.

En este estudio, el porcentaje global de cesáreas disminuyó de $44,9 \%$ a $37,1 \%$, y en nulíparas disminuyó desde $39,0 \%$ a $29,5 \%$ y en multíparas de 48,1 a $42,2 \%$, diferencias estadísticamente significativas. El índice de cesáreas en 177 nulíparas en trabajo de parto espontáneo con embarazo simple, de término y en cefálica se redujo desde $22,7 \%$ a $16,4 \%$ en un período previo de 3 meses, mientras que el índice de cesáreas en 253 multíparas en trabajo de parto espontáneo con embarazo simple, de término y en cefálica se redujo de $16,3 \%$ a $10,7 \%$, en igual período.

La base más adecuada para lograr cambios de conducta en la práctica clínica ha sido estudiada desde la perspectiva de las ciencias administrativas, conocido como efecto Hawthorne. Este establece que si un individuo o un grupo de personas están al tanto de ser observados, su conducta es propensa a cambiar. Esto pudo haber jugado un rol importante en lograr reducir los índices de cesáreas.

Si a la población objetivo, médicos y matronas, se le advierte de la dirección de un cambio deseado por sus observadores, a menudo ellos darán lo mejor de sí para cumplir los objetivos. Sin embargo, si actuó el efecto Hawthorne, se esperaría una extensa mejora en tasas de operación cesárea y no mejorías aisladas en subgrupos específicos. En este estudio, la significativa reducción de cesáreas en nulíparas con embarazo simple, en cefálica, de término y en trabajo de parto espontáneo, sugiere que algunos cambios del manejo obstétrico que fueron introducidos, como los plazos de las pruebas de trabajo de parto, el uso de ocitócicos, el momento para la anestesia de conducción y otros, fueron importantes.

Para prevenir la aclimatación a la observación, es fundamental que se realicen frecuentemente reuniones de revisión de casos clínicos, adecuadamente documentados y utilizados para monitorear cuidadosamente los cambios de la práctica obstétrica. Esto asegura que las prácticas que son cambiadas se mantengan, debido al reforzamiento que suele producir una observación continua. Se retrasa así la aclimatación a la observación, hasta que el feedback de la auditoría médica sobre la efectividad de las prácticas logre el consenso del cambio de conducta.

Se ha sugerido que la falta de consenso en la reducción de las tasas de cesáreas requiere de medidas más agresivas para implementar nuevas recomendaciones. En nuestro estudio, el índice de cesáreas se redujo de un nivel que muchos verían como razonable, ya que las cesáreas se realiza- 
ron en embarazos no complicados. Médicos y matronas se interesaron activamente en el manejo del trabajo de parto normal, lo que permitió reducir las cesáreas.

La mayor parte de esta reducción parece ser consecuencia de cambios específicos en el manejo del trabajo de parto espontáneo en mujeres nulíparas, disminuyendo así la incidencia de operación cesárea por distocias. Sin embargo, los autores reconocen que el uso de controles históricos en un estudio basado en auditoría, hace difícil excluir completamente otras variables "escondidas".

En Chile, las tasas de cesáreas, tanto en el sector público como privado alcanza niveles que lo ubica entre los países latinoamericanos con mayor prevalencia $(14,15)$, de ahí que la aplicación de auditorías similares, en otros entornos, podría identificar actitudes intervencionistas cuyos cambios podría determinar la reducción del índice de cesáreas. De hecho, si el índice de operación cesárea de una institución es mayor a nuestro $44,9 \%$ inicial, hace posible que dependiendo de los resultados de una auditoría local, el espacio correspondiente para la reducción podría ser mayor.

Los profesionales responsables del manejo de embarazos no complicados debieran tener un interés activo de poner en práctica los principios del ciclo de la auditoría médica, mientras sea aplicable en sus servicios. Por sobre todo, debemos intentar prevenir que los nacimientos no complicados se conviertan en complicados y por ende elevar la tasa de cesáreas en aquellos casos que pudieron ser "evitadas".

\section{BIBLIOGRAFIA}

1. Yudkin PL, Word L, Redman CW: Risk of unexplained stillbirth at different gestational ages. Lancet 1987; 1: 1192-94.
2. Notzon FC, Placek PJ, Taffel SM: Comparison of national caesarean section rates. $\mathrm{N}$ Engl $\mathrm{J}$ Med 1987; 316: 386-9.

3. Lilford RJ, Van Coeverden De Groot HA, Moore PJ, Bingham $P$ : The relative risks of caesarean section (intrapartum and elective) and vaginal delivery: a detailed análisis to exclude the effects of medical disorders and other pre-existing physiological disturbances. Br J Obstet Gynaecol 1990; 97: 88392.

4. O'Driscoll K, Foley M: Correlation of decrease in perinatal mortality and increase in caesarean section rates. Obstet Gynecol 1983; 61: 1-5.

5. Rasmussen S, Irgens LM, Dalaker K: A history of placental dysfunction and risk of placental abruption. Paediatr Perinat Epidemiol 1999; 13: 9-21.

6. Lydon-Rochelle M, Holt V, Easterling TR, Martin DP: First-birth cesarean and placental abruption or previa at second birth. Obstet Gynecol 2001; 97:76569.

7. Savage W, Francome C: British caesarean section rates: have we reached a plateau? $\mathrm{Br} J$ Obstet Gynaecol 1993; 100: 493-6.

8. Farmer A: Medical practice guidelines: lessons from the United States. BMJ 1993; 307: 313-7.

9. Kassirer JP: Clinical trials and meta-analysis: what do they do for us? N Engl J Med 1992; 327: 273-4.

10. Secretaries of State for Health Wales, Northern Ireland, and Scotland. Medical audit. London: Her Majesty's Stationery Office, 1989.

11. Fowkes FGR: Medical audit cycle: a review of methods and research in clinical practice. Med Educ 1982; 16: 228-38.

12. Smith T: Medical audit: closing the feedback loops is vital. BMJ 1990; 300: 65-9.

13. Robson MS, Scudamore IW, Walsh SM: Using the medical audit cycle to reduce cesarean section rates. Am J Obstet Gynecol 1996; 174(1): 199-205.

14. Belizan JM, Althabe F, Barros FC, Alexander S. Rates and implications of caesarean section in Latin America: ecological study. BMJ 1999; 319: 1397-402.

15. Murray SF. Relation between private health insurance and high rates of caesarean section in Chile: qualitative and quantitative study. BMJ 2000; 321: 1501-5. 